\title{
Global Finite-Time Set Stabilization of Spacecraft Attitude with Disturbances using Second-Order Sliding Mode Control
}

\section{Zeyu Guo}

Southeast University

Zuo Wang ( $\nabla$ z.wang@seu.edu.cn )

Southeast University

Shihua Li

Southeast University School of Automaton

\section{Research Article}

Keywords: spacecraft, attitude stabilization, global finite-time stability, second-order sliding mode, bounded disturbances

Posted Date: October 21st, 2021

DOI: https://doi.org/10.21203/rs.3.rs-997565/v1

License: (c) (i) This work is licensed under a Creative Commons Attribution 4.0 International License. Read Full License

Version of Record: A version of this preprint was published at Nonlinear Dynamics on February 3rd, 2022. See the published version at https://doi.org/10.1007/s11071-022-07245-5. 


\title{
Global Finite-Time Set Stabilization of Spacecraft Attitude with Disturbances using Second-Order Sliding Mode Control
}

\author{
Guo Zeyu ${ }^{1,2 \dagger}$, Wang Zuo ${ }^{1,2^{*}}$ and Li Shihua ${ }^{1,2 \dagger}$ \\ ${ }^{1}$ School of Automation, Southeast University, Nanjing, 210096, \\ PR, China. \\ ${ }^{2}$ Key Laboratory of Measurement and Control of Complex \\ Systems of Engineering, Ministry of Education, Nanjing, 210096, \\ PR, China.
}

Contributing authors: z.wang@seu.edu.cn;

$\dagger$ These authors contributed equally to this work.

\begin{abstract}
The performance of attitude stabilization control algorithms for rigid spacecraft can be limited by disturbances. In this paper, the global finite-time attitude stabilization problem with disturbances is investigated and handled by constructing a second-order sliding mode controller. Firstly, a virtual controller based on set stabilization idea is constructed to globally finite-time stabilize the system. Then, a relay polynomial second-order sliding mode controller is constructed to guarantee that the tracking error towards the virtual controller will converge to zero in finite-time. Finite-time Lyapunov theory is applied to support the proof and stability analysis. The global finite-time stability holds even with bounded disturbances. The effectiveness and feasibility of the controller are illustrated by the numerical simulations.
\end{abstract}

Keywords: spacecraft, attitude stabilization, global finite-time stability, second-order sliding mode, bounded disturbances 


\section{Introduction}

In recent years, the attitude stabilization control of the spacecraft has attracted extensive attentions due to its prominent role in space missions [1-3], such as spacecraft pointing, maneuvering, etc. It is well known that the linear controllers, e.g., the PID controllers [4-6], are widely adopted in spacecraft due to their simple structure and easy implementation. However, PID controllers are hard to satisfy the requirements of high performance owing to the existence of couplings, nonlinearities and disturbances in spacecraft dynamics. Thus, the ensuing research efforts are devoted to the nonlinear approaches. These available control schemes include, but not limited to, backstepping control $[7,8], H_{\infty}$ control $[9,10]$, inverse optimal control [11, 12], sliding mode control (SMC) $[13,14]$ and adaptive control $[15,16]$. These advanced control algorithms focus on improving the performance of spacecraft attitude control from different aspects. Worth mentioning, the afore-mentioned methods only derive the asymptotic stability of the system, which means that the convergence time of the system states to the origin is infinite. In order to improve the dynamic performance of the attitude control system, finite-time control schemes have attracted the investigate attentions from researchers.

Results have shown that faster dynamic responses, higher steady-state accuracies, and better robust performances can be achieved by applying finitetime control approaches as compared with the corresponding asymptotically stable control approaches $[17,18]$. After years of research, significant progresses have been made in finite-time control, during which numerous satisfactory approaches have been presented. These approaches can be divided into the following categories: the homogeneous control [19, 20], the adding a power integrator control (APIC) [21, 22], the terminal sliding mode control (TSMC) [23] and the higher-order sliding mode control (HOSM) [24]. However, there exist some limitations when homogeneous control and APIC are applied in the attitude control problems. For the homogeneous controller proposed in [19], disturbances can not be suppressed in the spacecraft dynamics. For the APIC approach in [21], only the finite-time boundedness can be ensured when dealing with disturbances.

Among the algorithms investigated for finite-time attitude stabilization, sliding mode control approaches have been proved to be effective owning to their robustness to disturbances. In [25], a TSMC method is proposed and the system states can be stabilized in finite time, while the unexpected singularity problem may occur in the implementation. In order to handle this problem, two different nonsingular TSMC (NTSMC) methods are proposed in $[26,27]$. To accelerate the convergence rates when the system states are far from the equilibrium, the fast NTSMC is proposed in [28]. Moreover, in [29], a fixedtime SMC is presented and the convergence time is independent of the initial states. Apart from the methods mentioned above, there exist various extensions of TSMC to improve the control performance from different aspects, e.g., observer-based output feedback TSMC [30-32], actuator saturated TSMC $[33,34]$, adaptive TSMC $[35,36]$, etc. In addition, the discontinuous control 
inputs lead to the chattering phenomenon. To this end, HOSM methods are applied in spacecraft to address chattering and guarantee the finite-time convergence as well. In [37], a integral second-order SMC (SOSM) is constructed, by which the finite-time stability of the system was guaranteed. On this basis, a third-order SMC method is proposed in in [38]. By hiding the discontinuous switching in the derivative of the control inputs, the continuous control inputs are derived. However, both of the above two papers involve the inverse of a time-varying matrix, which may be irreversible in some specific states, thus the singularity problem may occur.

To sum up, the aforementioned papers provide various finite-time control methods for the attitude stabilization of spacecraft. However, there still exists one main problem that the results are not global. It is well known that the quaternion is usually used to describe the arbitrary attitude motion in the three-dimensional space globally. The attitude systems under quaternionbased descriptions all have two equilibria. For these systems, the stability involved is called set stability and its definition can be consulted in [39, 40]. For the literatures using quaternion-based attitude descriptions, the global result means that the system is set stable, specifically, both of the two equilibria should be designed to be stable. If only one of the equilibria is designed to be stable while the other equilibrium is designed to be unstable, the attractive domain of the stable equilibrium will cover the global state space excluding one particular point. In this case, the entire system states will converge to the stable equilibrium although some states are closer to the unstable one, which is called 'unwinding' phenomenon [41]. Hence, the controllers that only consider one equilibrium all fail to derive set stability and their results can only be considered as almost global results like that in [42].

The global attitude stabilization methods can be seen in [43-45] and the references therein. In [43], the idea of set stability is combined with the SOSM to obtain global stability. However, since the sliding mode surface selected in this paper is linear, the system is asymptotically stable. In [44], an adaptive tripodal hybrid control scheme is raised to globally stabilize the system, but the system can not ensure the finite-time convergence as well. In [45], by applying the set stabilization idea, the system states will converge to different equilibria depending on the initial states, however, only in the absence of disturbances. There are fewer controllers which can obtain global finite-time results with disturbances. Therefore, the motivation of this investigation is to design a controller which can derive global finite-time stability of the system when dealing with disturbances.

In this paper, inspired by the set stabilization idea, a relay polynomial SOSM (RPSOSM) controller will be constructed by using a backstepping-like way to handle the global finite-time attitude stabilization problem. Firstly, a virtual controller will be constructed to globally stabilize the system. Then the SOSM controller will be designed. The finite-time tracking towards the virtual controller and the global finite-time stability of the system will be proved based on Lyapunov theory. The major remarkable features are as follows: 
1) A set stabilization law is applied to obtain a global result in the stabilization of spacecraft attitude.

2) When dealing with the disturbances, the system states still converge to the equilibrium in finite-time.

3) The structure of the designed controller is simple and only one parameter needs to be tuned.

The rest of the paper can be summarized as follows. In Section 2, we will present some lemmas, after which the dynamic and kinematic model of spacecraft attitude will be provided. Also, the problem formulation will be established at the end of the section. In Section 3, we will give the design scheme of the controller as well as the stability analysis of the closed-loop system. In Section 4, simulation results are displayed and analyzed. The conclusion part will be included in Section 5 .

\section{System model and problem formulation}

\subsection{Preliminaries}

The expression and lemmas involved in subsequent derivations and proofs are listed here. For simplicity, we use the notation $\lfloor x\rceil^{\alpha}=\operatorname{sign}(x)|x|^{\alpha}, x \in R$.

Definition $1[39,46]$ : Suppose there exists a compact set $M$ and a continuous function $V(\boldsymbol{x}): R^{n} \rightarrow[0,+\infty)$. Then the $V(x)$ is said to be a positive definite function with respect to $M$ if $\forall x \in R^{n} \backslash M, V(\boldsymbol{x})>0$ and $V(M)=0$.

Lemma 1 [17]: For the following system

$$
\dot{x}=f(x), \quad f(0)=0, \quad x \in R^{n}
$$

suppose there exists a positive definite function $V(x): U \rightarrow R$ such that the following condition holds

(i) There exists real numbers $c>0, \alpha \in(0,1)$ and an open neighbourhood $U_{0} \subset U$ of the origin such that

$$
\dot{V}(x)+c V^{\alpha}(x) \leq 0, \quad x \in U_{0} \backslash\{0\}
$$

Then the origin is a finite-time stable equilibrium of system (1). If $U=U_{0}=R^{n}$, the origin is a global finite-time stable equilibrium of system (1).

Lemma 2 [47]: If $p_{1}>0$ and $0<p_{2} \leq 1$, then for $\forall x, y \in R$, the following inequality holds

$$
\left|\lfloor x\rceil^{p_{1} p_{2}}-\lfloor y\rceil^{p_{1} p_{2}}\right| \leq 2^{1-p_{2}}\left|\lfloor x\rceil^{p_{1}}-\lfloor y\rceil^{p_{1}}\right|^{p_{2}} .
$$


Lemma 3 [48]: Let $c$ and $d$ be positive constants, then $\forall x, y \in R$ satisfy the inequality

$$
|x|^{c}|y|^{d} \leq \frac{c}{c+d}|x|^{c+d}+\frac{d}{c+d}|y|^{c+d}, \forall x, y \in R .
$$

Lemma 4 [49]: Let $p$ be a real number with $0<p<1$, then for $\forall x_{i} \in R, i=$ $1, \ldots, n$, we have

$$
\left(\left|x_{1}\right|+\cdots\left|x_{n}\right|\right)^{p} \leq\left|x_{1}\right|^{p}+\cdots+\left|x_{n}\right|^{p}
$$

Lemma 5 [50]: Let $V(x)$ be a continuous positive-definite function with respect to a compact set $M$ for system(1). If $V(x)$ satisfies $\dot{V}(x) \leq 0, \forall x \in$ $R^{n} \backslash M$, then system (1) is stable with respect to $M$.

\subsection{Attitude model and problem formulation}

For the attitude stabilization problems, the dynamic model of spacecraft attitude can be described as [51]

$$
J \dot{\omega}=a(\omega) J \omega+u(t)+M(t)
$$

where $\boldsymbol{J}=\boldsymbol{J}^{\boldsymbol{T}}$ is a positive-definite square inertia matrix whose dimension is $3, \boldsymbol{\omega}=\left[\omega_{1}, \omega_{2}, \omega_{3}\right]^{T}$ is the measured angular velocity column vector, and $\boldsymbol{u}(\boldsymbol{t})=\left[u_{1}, u_{2}, u_{3}\right]^{T}$ is the control torque column vector. $\boldsymbol{M}(\boldsymbol{t})=\left[M_{1}, M_{2}, M_{3}\right]^{T}$ is the disturbance column vector, e.g., solar radiation, magnetic effects or other uncertainties, which satisfies the following assumption:

Assumption 1: The disturbances in (2) are bounded by a know constant, which is $\sup \left|M_{i}(t)\right| \leq L, i=1,2,3$.

And $\boldsymbol{a}(\boldsymbol{\omega})$ is a $3 \times 3$ skew-symmetric matrix

$$
\boldsymbol{a}(\boldsymbol{\omega})=\left[\begin{array}{ccc}
0 & \omega_{3} & -\omega_{2} \\
-\omega_{3} & 0 & \omega_{1} \\
\omega_{2} & -\omega_{1} & 0
\end{array}\right]
$$

Using above dynamic model, with the quaternion being used to describe the spacecraft attitude, the kinematic model is [52]

$$
\dot{\boldsymbol{q}}=\frac{1}{2} \boldsymbol{E}(\boldsymbol{q}) \boldsymbol{\omega} .
$$


The quaternion $\boldsymbol{q}=\left[q_{0}, q_{v}^{T}\right]^{T}, \boldsymbol{q}_{\boldsymbol{v}}=\left[q_{1}, q_{2}, q_{3}\right]^{T}$ in (3) is the unit quaternion, it satisfies

$$
q_{0}=\cos \left(\frac{\theta}{2}\right), \quad q_{i}=e_{i} \sin \left(\frac{\theta}{2}\right), \quad i=1,2,3,
$$

where $\theta$ denotes the principal angle and $e=\left[e_{1}, e_{2}, e_{3}\right]^{T}$ denotes the Euler axis with $e_{1}^{2}+e_{2}^{2}+e_{3}^{2}=1$. Thus, the unit quaternion satisfies

$$
q_{0}^{2}+q_{1}^{2}+q_{2}^{2}+q_{3}^{2}=1 .
$$

$\boldsymbol{E}(\boldsymbol{q})$ is a $4 \times 3$ matrix

$$
\boldsymbol{E}(\boldsymbol{q})=\left(\begin{array}{c}
-\boldsymbol{q}_{v}^{T} \\
-\boldsymbol{a}\left(\boldsymbol{q}_{v}\right)+q_{0} \boldsymbol{I}_{\mathbf{3} \times \mathbf{3}}
\end{array}\right),
$$

where $\boldsymbol{I}_{\mathbf{3} \times \mathbf{3}}$ represents a $3 \times 3$ identity matrix. Based on (5), one can easily obtain that

$$
E^{T}(q) E(q)=I_{3 \times 3} .
$$

Using the nominal value of $\boldsymbol{J}$ to counteract the structural items in (2) and letting $\boldsymbol{u}(\boldsymbol{t})=\left[u_{1}, u_{2}, u_{3}\right]^{T}=-\boldsymbol{a}(\boldsymbol{w}) \boldsymbol{J} \boldsymbol{w}+\boldsymbol{J} \boldsymbol{u}_{\boldsymbol{s}}(\boldsymbol{t})$, the system model can be simplified to

$$
\begin{aligned}
\dot{\boldsymbol{q}} & =\frac{1}{2} \boldsymbol{E}(\boldsymbol{q}) \boldsymbol{\omega}, \\
\dot{\boldsymbol{\omega}} & =\boldsymbol{u}_{\boldsymbol{s}}+\boldsymbol{d}(\boldsymbol{t}),
\end{aligned}
$$

where $\boldsymbol{u}_{\boldsymbol{s}}(\boldsymbol{t})=\left[u_{s 1}, u_{s 2}, u_{s 3}\right]^{T}$ denotes the simplified control law. By Assumption $1, \boldsymbol{d}(\boldsymbol{t})=\boldsymbol{J}^{-1} \boldsymbol{M}(\boldsymbol{t})=\left[d_{1}(t), d_{2}(t), d_{3}(t)\right]^{T}$ is also bounded by a known positive constant.

As presented in (4) and (5), to derive set stability, there should be two equilibria $(1,0,0,0)^{T}$ and $(-1,0,0,0)^{T}$. The difference in principal angle between them is $360^{\circ}$, and it seems to be exactly the same from a geometric point of view, based on which many papers claim themselves global stable though they only consider one equilibrium. Obviously, this is not entirely true for the reason that if the equilibrium $(1,0,0,0)^{T}$ is designed to be stable while $(-1,0,0,0)^{T}$ is unstable, the entire system states will converge to the equilibrium $(1,0,0,0)^{T}$ although some states are closer to $(-1,0,0,0)^{T}$, engendering the so-called 'unwinding' phenomenon. To avoid this phenomenon, a SOSM control scheme based on set stability is introduced in subsequent sections.

\section{Second-order sliding mode controller design}

In this section, a SOSM controller is designed by using the idea of APIC and set stability. The design process and stability analysis are divided into two steps.

At first, we select the sliding variables as follows:

$$
\begin{aligned}
& s_{0}=q_{0}-\operatorname{sign}\left(q_{0}(0)\right), \\
& s_{i}=q_{i}, i=1,2,3,
\end{aligned}
$$


where $q_{0}(0)$ denotes the initial condition of $q_{0}$.

Theorem 1: Considering the system (7) with Assumption 1, there exists positive constants $k_{1}, k_{2}$ such that

$$
u_{s i}=-k_{1} \operatorname{sign}\left(\left\lfloor\omega_{i}\right\rceil^{2}+\operatorname{sign}\left(q_{0}(0)\right) k_{2}{ }^{2}\left\lfloor s_{i}\right\rceil\right), i=1,2,3
$$

the SOSM $s_{i}=\dot{s}_{i}=0, i=0,1,2,3$ will be established in finite time.

Remark 1: As the $q_{0}$ is always available throughout the control process, the value of $q_{0}(0)$ can be obtained directly. By introducing the sign $\left(q_{0}(0)\right)$ term into the sliding variable, it will be proved later that whether the system states converge to whether the system converges to $(1,0,0,0)^{T}$ or $(-1,0,0,0)^{T}$ depends on the initial value of $q_{0}$. In other words, if the above sliding variables can be driven to the origin in finite time, the global finite-time stability can be achieved.

Proof. Step 1: Choosing a $C^{1}$ Lyapunov function of the form:

$$
V_{1}(\boldsymbol{s})=s_{0}^{2}+s_{1}^{2}+s_{2}^{2}+s_{3}^{2}=\left[q_{0}-\operatorname{sign}\left(q_{0}(0)\right)\right]^{2}+\boldsymbol{q}_{\boldsymbol{v}}^{\boldsymbol{T}} \boldsymbol{q}_{\boldsymbol{v}} .
$$

Then taking derivative of (10) along system (7) provides

$$
\begin{aligned}
\dot{V}_{1}(\boldsymbol{s}) & =2\left(q_{0}-\operatorname{sign}\left(q_{0}(0)\right)\right) \dot{q}_{0}+2 \boldsymbol{q}_{\boldsymbol{v}}^{\boldsymbol{T}} \dot{\boldsymbol{q}}_{\boldsymbol{v}} \\
& =\operatorname{sign}\left(q_{0}(0)\right) \boldsymbol{q}_{\boldsymbol{v}}^{\boldsymbol{T}} \boldsymbol{\omega} \\
& =\operatorname{sign}\left(q_{0}(0)\right) \boldsymbol{q}_{\boldsymbol{v}}^{\boldsymbol{T}} \boldsymbol{\omega}^{*}+\operatorname{sign}\left(q_{0}(0)\right) \boldsymbol{q}_{\boldsymbol{v}}^{\boldsymbol{T}}\left(\boldsymbol{\omega}-\boldsymbol{\omega}^{*}\right) .
\end{aligned}
$$

$\boldsymbol{\omega}^{*}=\left[\omega_{1}^{*}, \omega_{2}^{*}, \omega_{3}^{*}\right]^{T}$ is the virtual control law which is designed as

$$
\omega_{i}^{*}=-\operatorname{sign}\left(q_{0}(0)\right) k_{2}\left\lfloor q_{i}\right\rceil^{\frac{1}{2}}, i=1,2,3 .
$$

Using Lemma 4, supposing that the tracking error towards the virtual controller has converged to zero in finite-time and considering the condition that $q_{0}(0) \geq 0$, then $(11)$ can be expressed as

$$
\begin{aligned}
\dot{V}_{1}(s) & =-k_{2}\left(\left|q_{1}\right|^{\frac{3}{2}}+\left|q_{2}\right|^{\frac{3}{2}}+\left|q_{3}\right|^{\frac{3}{2}}\right) \\
& \leq-k_{2}\left(q_{1}^{2}+q_{2}^{2}+q_{3}^{2}\right)^{\frac{3}{4}} \\
& =-k_{2}\left(1-q_{0}^{2}\right)^{\frac{3}{4}}
\end{aligned}
$$

From system (7) we have $\dot{q}_{0}=-\frac{1}{2}\left(q_{1} \omega_{1}+q_{2} \omega_{2}+q_{3} \omega_{3}\right)$. This, together with virtual control law (12), implies

$$
\dot{q}_{0}=\frac{1}{2} k_{2}\left(\left|q_{1}\right|^{\frac{3}{2}}+\left|q_{2}\right|^{\frac{3}{2}}+\left|q_{3}\right|^{\frac{3}{2}}\right) .
$$


As $k_{2}$ is a positive constant, the condition $\dot{q}_{0}>0$ and $q_{0}>0$ will be reached in a finite time $t_{1}$. This fact leads to

$$
\begin{aligned}
\dot{V}_{1}(s) & =-k_{2}\left(\left|q_{1}\right|^{\frac{3}{2}}+\left|q_{2}\right|^{\frac{3}{2}}+\left|q_{3}\right|^{\frac{3}{2}}\right) \\
& \leq-k_{2}\left(1-q_{0}^{2}\right)^{\frac{3}{4}} \\
& =-k_{2}\left(1-q_{0}\right)^{\frac{3}{4}}\left(1+q_{0}\right)^{\frac{3}{4}} \\
& \leq-\frac{k_{2}}{2}\left(2-2 q_{0}\right)^{\frac{3}{4}} .
\end{aligned}
$$

Another fact is $V_{1}(\boldsymbol{s})=\left(q_{0}-1\right)^{2}+\boldsymbol{q}_{\boldsymbol{v}}^{\boldsymbol{T}} \boldsymbol{q}_{\boldsymbol{v}}=2-2 q_{0}$. This, together with (15), one has

$$
\dot{V}_{1}(s)+\frac{k_{2}}{2} V_{1}^{\frac{3}{4}}(s) \leq 0, t \geq t_{1} .
$$

The same proof turns out to be correct for the case $q_{0}(0)<0$. Using Lemma 1 , it is not complicated to conclude that system (7) under the designed virtual controller (12) is globally finite-time stable.

One may consider that, based on backstepping control, a controller that drives the tracking error to 0 in finite time can be constructed directly. However, the derivative operation in the design process will bring the control input with the negative fractional power, which may cause singularity problem. For this reason, we use a generalized 'APIC' [53] to guarantee the finite-time tracking of the virtual controller and the establishment of SOSM.

Substituting (12) into (11), it yields

$$
\begin{aligned}
\dot{V}_{1}(\boldsymbol{s}) & =-k_{2}\left(q_{1}\left\lfloor q_{1}\right\rceil^{\frac{1}{2}}+q_{2}\left\lfloor q_{2}\right\rceil^{\frac{1}{2}}+q_{3}\left\lfloor q_{3}\right\rceil^{\frac{1}{2}}\right)+\operatorname{sign}\left(q_{0}(0)\right) \boldsymbol{q}_{\boldsymbol{v}}^{\boldsymbol{T}}\left(\boldsymbol{\omega}-\boldsymbol{\omega}^{*}\right) \\
& =-k_{2}\left(\left|q_{1}\right|^{\frac{3}{2}}+\left|q_{2}\right|^{\frac{3}{2}}+\left|q_{3}\right|^{\frac{3}{2}}\right)+\operatorname{sign}\left(q_{0}(0)\right) \boldsymbol{q}_{\boldsymbol{v}}^{\boldsymbol{T}}\left(\boldsymbol{\omega}-\boldsymbol{\omega}^{*}\right)
\end{aligned}
$$

We know sign $\left(q_{0}(0)\right) \boldsymbol{q}_{\boldsymbol{v}}^{\boldsymbol{T}}\left(\boldsymbol{w}-\boldsymbol{w}^{*}\right) \leq\left|q_{1}\right|\left|w_{1}-w_{1}^{*}\right|+\left|q_{2}\right|\left|w_{2}-w_{2}^{*}\right|+\left|q_{3}\right|\left|w_{3}-w_{3}^{*}\right|$. Denoting $\left|\omega_{i}-\omega_{i}^{*}\right|, i=1,2,3$ as $\left|\left\lfloor\omega_{i}\right\rceil^{2 \times \frac{1}{2}}-\left\lfloor\omega_{i}^{*}\right\rceil^{2 \times \frac{1}{2}}\right|$ and using Lemma 2 , it provides

$$
\left|q_{i}\right|\left|w_{i}-w_{i}^{*}\right| \leq \sqrt{2}\left|q_{i}\right|\left|\delta_{i}\right|^{\frac{1}{2}}, i=1,2,3,
$$

where $\delta_{i}=\left\lfloor\omega_{i}\right\rceil^{2}-\left\lfloor\omega_{i}^{*}\right\rceil^{2}$. Applying Lemma 3 to (18), it yields

$$
\begin{aligned}
\left|q_{i}\right|\left|w_{i}-w_{i}^{*}\right| & \leq \sqrt{2}\left|q_{i}\right|\left|\delta_{i}\right|^{\frac{1}{2}} \\
& \leq \sqrt{2}\left(\frac{2}{3}\left|q_{i}\right|^{\frac{3}{2}}+\frac{1}{3}\left|\delta_{i}\right|^{\frac{3}{2}}\right), i=1,2,3 .
\end{aligned}
$$

Then combining (17) and (19) leads to

$$
\dot{V}_{1}(s) \leq\left(-k_{2}+\frac{2 \sqrt{2}}{3}\right)\left(\left|q_{1}\right|^{\frac{3}{2}}+\left|q_{2}\right|^{\frac{3}{2}}+\left|q_{3}\right|^{\frac{3}{2}}\right)+\frac{\sqrt{2}}{3}\left(\left|\delta_{1}\right|^{\frac{3}{2}}+\left|\delta_{2}\right|^{\frac{3}{2}}+\left|\delta_{3}\right|^{\frac{3}{2}}\right) .
$$


Step 2: Considering the tracking error between $\boldsymbol{\omega}$ and $\boldsymbol{\omega}^{*}$, and defining $V_{i}(\boldsymbol{s}, \boldsymbol{\omega})=\frac{4}{3 \sqrt{2} k_{2}^{3}} \int_{\omega_{i}^{*}}^{\omega_{i}}\left\lfloor\lfloor k\rceil^{2}-\left\lfloor\omega_{i}^{*}\right\rceil^{2}\right\rceil^{\frac{3}{2}} d k, i=1,2,3$, based on which we can choose a $C^{1}$ Lyapunov function

$$
V(\boldsymbol{s}, \boldsymbol{\omega})=V_{1}(\boldsymbol{s})+V_{1}(\boldsymbol{s}, \boldsymbol{\omega})+V_{2}(\boldsymbol{s}, \boldsymbol{\omega})+V_{3}(\boldsymbol{s}, \boldsymbol{\omega}) .
$$

Since $V_{1}(s)$ and $V_{i}(s, \boldsymbol{\omega})$ are positive definite, $V(\boldsymbol{s}, \boldsymbol{\omega})$ is also positive definite. According to Definition $1, V(\boldsymbol{s}, \boldsymbol{\omega})$ is a Lyapunov function with respect to set M for system (7) where $M=\left\{\left(\boldsymbol{q}^{\boldsymbol{T}}, \boldsymbol{\omega}^{\boldsymbol{T}}\right) \mid(-1,0,0,0,0,0,0),(1,0,0,0,0,0,0)\right\}$. Taking the derivative of $V_{i}(\boldsymbol{s}, \boldsymbol{\omega})$ along the system we have

$$
\dot{V}_{i}(\boldsymbol{s}, \boldsymbol{w})=-\frac{\sqrt{2}}{k_{2}^{3}} \frac{\left(\partial\left\lfloor w_{i}^{*}\right\rceil^{2}\right)}{\partial q_{i}} \dot{q}_{i} \int_{\omega_{i}^{*}}^{\omega_{i}}\left\lfloor\lfloor k\rceil^{2}-\left\lfloor\omega_{i}^{*}\right\rceil^{2}\right\rceil^{\frac{1}{2}} d k+\frac{4}{3 \sqrt{2} k_{2}^{3}}\left\lfloor\delta_{i}\right\rceil^{\frac{3}{2}}\left(u_{s i}+d_{i}\right) .
$$

Now we we analyze each of the terms on the right-hand side of (22). To begin with, The inequalities $\dot{q}_{i} \leq\left|\dot{q}_{i}\right| \leq \frac{1}{2}\left(\left|\omega_{1}\right|+\left|\omega_{2}\right|+\left|\omega_{3}\right|\right)$ and $\int_{\omega_{i}^{*}}^{\omega_{i}}\left\lfloor\lfloor k\rceil^{2}-\right.$ $\left.\left\lfloor\omega_{i}^{*}\right\rceil^{2}\right\rceil^{\frac{1}{2}} d k \leq\left|\omega_{i}-\omega_{i}^{*}\right|\left|\delta_{i}\right|^{\frac{1}{2}}$ hold obviously. Thus, (22) can be rewritten as

$$
\begin{aligned}
\dot{V}_{i}(\boldsymbol{s}, \boldsymbol{w}) & \leq \frac{\sqrt{2}}{2 k_{2}^{3}}\left|\frac{\left(\partial\left\lfloor w_{i}^{*}\right\rceil^{2}\right)}{\partial q_{i}}\right|\left(\left|w_{1}\right|+\left|w_{2}\right|+\left|w_{3}\right|\right)\left|\omega_{i}-\omega_{i}^{*}\right|\left|\delta_{i}\right|^{\frac{1}{2}} \\
& +\frac{4}{3 \sqrt{2} k_{2}^{3}}\left\lfloor\delta_{i}\right\rceil^{\frac{3}{2}}\left(u_{s i}+d_{i}\right) .
\end{aligned}
$$

According to (12), one has

$$
\left|\frac{\left(\partial\left\lfloor w_{i}^{*}\right\rceil^{2}\right)}{\partial q_{i}}\right|=k_{2}^{2}
$$

This, together with (23), it yields

$$
\dot{V}_{i}(\boldsymbol{s}, \boldsymbol{w}) \leq \frac{\sqrt{2}}{2 k_{2}}\left(\left|w_{1}\right|+\left|w_{2}\right|+\left|w_{3}\right|\right)\left|\omega_{i}-\omega_{i}^{*}\right|\left|\delta_{i}\right|^{\frac{1}{2}}+\frac{4}{3 \sqrt{2} k_{2}^{3}}\left\lfloor\left.\delta_{i}\right|^{\frac{3}{2}}\left(u_{s i}+d_{i}\right) .\right.
$$

In (25), the term $\left|\omega_{i}-\omega_{i}^{*}\right|\left|\delta_{i}\right|^{\frac{1}{2}}\left|\omega_{j}\right|$ can be enlarged further. Using Lemma 2, we have

$$
\left|\omega_{i}-\omega_{i}^{*}\right|\left|\delta_{i}\right|^{\frac{1}{2}}\left|\omega_{j}\right| \leq \sqrt{2}\left|\delta_{i}\right|\left|\omega_{j}\right| \text {. }
$$

Denoting $\left|\omega_{j}\right|$ as $\left|\omega_{j}^{*}+\omega_{j}-\omega_{j}^{*}\right|$ and applying the inequality $\|\boldsymbol{x}+\boldsymbol{y}\| \leq\|\boldsymbol{x}\|$ $+\|\boldsymbol{y}\|$, it provides

$$
\left|\omega_{i}-\omega_{i}^{*}\right|\left|\delta_{i}\right|^{\frac{1}{2}}\left|\omega_{j}\right| \leq \sqrt{2}\left|\delta_{i}\right|\left|\omega_{j}^{*}\right|+\sqrt{2}\left|\delta_{i}\right|\left|\omega_{j}-\omega_{j}^{*}\right|
$$


Based on Lemma 2, we have $\left|\omega_{j}-\omega_{j}^{*}\right| \leq \sqrt{2}\left|\delta_{j}\right|^{\frac{1}{2}}$. Substituting it into (27) and applying Lemma 3, it yields

$$
\left|\omega_{i}-\omega_{i}^{*}\right|\left|\delta_{i}\right|^{\frac{1}{2}}\left|\omega_{j}\right| \leq \sqrt{2}\left|\delta_{i}\right|\left|\omega_{j}^{*}\right|+\sqrt{2}\left(\frac{2}{3}\left|\delta_{i}\right|^{\frac{3}{2}}+\frac{1}{3}\left|\delta_{j}\right|^{\frac{3}{2}}\right) .
$$

This, together with (12) and Lemma 3, it implies

$$
\left|\omega_{i}-\omega_{i}^{*}\right|\left|\delta_{i}\right|^{\frac{1}{2}}\left|\omega_{j}\right| \leq \sqrt{2} k_{2}\left(\frac{2}{3}\left|\delta_{i}\right|^{\frac{3}{2}}+\frac{1}{3}\left|q_{j}\right|^{\frac{3}{2}}\right)+\left(\frac{4}{3}\left|\delta_{i}\right|^{\frac{3}{2}}+\frac{2}{3}\left\lfloor\left.\delta_{j}\right|^{\frac{3}{2}}\right) .\right.
$$

Substituting (29) into (25) and simplifying it, we have

$$
\begin{aligned}
\dot{V}_{i}(\boldsymbol{s}, \boldsymbol{w}) & \leq\left[\left(\frac{2 \sqrt{2}}{k_{2}}+2\right)\left|\delta_{i}\right|^{\frac{3}{2}}+\frac{\sqrt{2}}{3 k_{2}}\left(\left|\delta_{1}\right|^{\frac{3}{2}}+\left|\delta_{2}\right|^{\frac{3}{2}}+\left|\delta_{3}\right|^{\frac{3}{2}}\right)\right. \\
& \left.+\frac{1}{3}\left(\left|q_{1}\right|^{\frac{3}{2}}+\left|q_{2}\right|^{\frac{3}{2}}+\left|q_{3}\right|^{\frac{3}{2}}\right)\right]+\frac{4}{3 \sqrt{2} k_{2}^{3}}\left\lfloor\left.\delta_{i}\right|^{\frac{3}{2}}\left(u_{s i}+d_{i}\right) .\right.
\end{aligned}
$$

Substituting (20) and (30) into the derivative of (21), it yields

$$
\begin{aligned}
\dot{V}(s, \boldsymbol{w}) & =\dot{V}_{1}(\boldsymbol{s})+\dot{V}_{1}(\boldsymbol{s}, \boldsymbol{w})+\dot{V}_{2}(\boldsymbol{s}, \boldsymbol{w})+\dot{V}_{3}(\boldsymbol{s}, \boldsymbol{w}) \\
& \leq\left(-k_{2}+\frac{2 \sqrt{2}}{3}+1\right)\left(\left|q_{1}\right|^{\frac{3}{2}}+\left|q_{2}\right|^{\frac{3}{2}}+\left|q_{3}\right|^{\frac{3}{2}}\right) \\
& +\left(\frac{\sqrt{2}}{3}+\frac{3 \sqrt{2}}{k_{2}}+2\right)\left(\left|\delta_{1}\right|^{\frac{3}{2}}+\left|\delta_{2}\right|^{\frac{3}{2}}+\left|\delta_{3}\right|^{\frac{3}{2}}\right) \\
& +\frac{4}{3 \sqrt{2} k_{2}^{3}}\left(\left\lfloor\delta_{1}\right\rceil^{\frac{3}{2}} u_{s 1}+\left\lfloor\delta_{1}\right\rceil^{\frac{3}{2}} d_{1}+\left\lfloor\delta_{2}\right\rceil^{\frac{3}{2}} u_{s 2}+\right. \\
& \left.\left\lfloor\delta_{2}\right\rceil^{\frac{3}{2}} d_{2}+\left\lfloor\delta_{3}\right\rceil^{\frac{3}{2}} u_{s 3}+\left\lfloor\delta_{3}\right\rceil^{\frac{3}{2}} d_{3}\right) .
\end{aligned}
$$

Base on Assumption 1, let a positive constant $D$ denote the upper bound of the disturbances $d(t)$ such that $\sup \left|d_{i}(t)\right| \leq D, t \in[0,+\infty), i=1,2,3$ and represent (31) as

$$
\begin{aligned}
\dot{V}(s, \boldsymbol{w}) & \leq\left(-k_{2}+\frac{2 \sqrt{2}}{3}+1\right)\left(\left|q_{1}\right|^{\frac{3}{2}}+\left|q_{2}\right|^{\frac{3}{2}}+\left|q_{3}\right|^{\frac{3}{2}}\right) \\
& +\left(\frac{\sqrt{2}}{3}+\frac{3 \sqrt{2}}{k_{2}}+2+\frac{4 D}{3 \sqrt{2} k_{2}^{3}}\right)\left(\left|\delta_{1}\right|^{\frac{3}{2}}+\left|\delta_{2}\right|^{\frac{3}{2}}+\left|\delta_{3}\right|^{\frac{3}{2}}\right) \\
& +\frac{4}{3 \sqrt{2} k_{2}^{3}}\left(\left\lfloor\delta_{1}\right\rceil^{\frac{3}{2}} u_{s 1}+\left\lfloor\delta_{2}\right\rceil^{\frac{3}{2}} u_{s 2}+\left\lfloor\left.\delta_{3}\right|^{\frac{3}{2}} u_{s 3}\right) .\right.
\end{aligned}
$$


Letting $k_{3}$ denote a positive constant and another positive constant $k_{2}$ satisfy $k_{2}>k_{3}+1+\frac{2 \sqrt{2}}{3}$, then the controller can be designed as

$$
u_{s i}=-k_{1} \operatorname{sign}\left(\delta_{i}\right), i=1,2,3,
$$

where $k_{1}$ is also a positive constant which satisfies $k_{1}>\frac{3 \sqrt{2}}{4} k_{2}{ }^{3}\left(k_{3}+\frac{\sqrt{2}}{3}+\right.$ $\left.\frac{3 \sqrt{2}}{k_{2}}+2+\frac{4 D}{3 \sqrt{2} k_{2}^{3}}\right)$.

Remark 2: As mentioned in the introduction, the controller designed in this paper owns a simple structure and only one parameter needs to be tuned. From (33), it can be concluded that only parameter $k_{3}$ needs to be selected because the parameter $k_{2}$ is totally decided by $k_{3}$, while $k_{1}$ is decided by $k_{2}$ and $k_{3}$. Actually, there is even no parameter needs to be tuned because we can just let $k_{3}$ be a small positive constant like 0.01 . Thus, it is reasonable to claim that the controller in this paper are much more simpler than others.

Substituting control law (33) into (32), it yields

$$
\dot{V}(\boldsymbol{s}, \boldsymbol{w}) \leq-k_{3}\left(\left|q_{1}\right|^{\frac{3}{2}}+\left|q_{2}\right|^{\frac{3}{2}}+\left|q_{3}\right|^{\frac{3}{2}}\right)-k_{3}\left(\left|\delta_{1}\right|^{\frac{3}{2}}+\left|\delta_{2}\right|^{\frac{3}{2}}+\left|\delta_{3}\right|^{\frac{3}{2}}\right) .
$$

On the basis of Lemma 5, it can be derived from (34) that system (7) under the controller (33) is stable with respect to set $M=$ $\left\{\left(\boldsymbol{q}^{\boldsymbol{T}}, \boldsymbol{\omega}^{\boldsymbol{T}}\right) \mid(-1,0,0,0,0,0,0),(1,0,0,0,0,0,0)\right\}$. Thus, the set stability of the system has been proved. Then we go on to prove that the convergence time of the system states is finite. We know $V(\boldsymbol{s}, \boldsymbol{\omega})$ satisfies

$$
\begin{aligned}
V(\boldsymbol{s}, \boldsymbol{\omega}) & =\left[q_{0}-\operatorname{sign}\left(q_{0}(0)\right)\right]^{2}+{q_{1}}^{2}+{q_{2}}^{2}+{q_{3}}^{2}+V_{1}(\boldsymbol{s}, \boldsymbol{\omega})+V_{2}(\boldsymbol{s}, \boldsymbol{\omega})+V_{3}(\boldsymbol{s}, \boldsymbol{\omega}) \\
& \leq\left[q_{0}-\operatorname{sign}\left(q_{0}(0)\right)\right]^{2}+{q_{1}}^{2}+{q_{2}}^{2}+{q_{3}}^{2}+\frac{4}{3 \sqrt{2}{k_{2}}^{3}}\left|w_{1}-w_{1}{ }^{*}\right|\left|\delta_{1}\right|^{\frac{3}{2}} \\
& +\frac{4}{3 \sqrt{2} k_{2}{ }^{3}}\left|w_{2}-w_{2}{ }^{*}\right|\left|\delta_{2}\right|^{\frac{3}{2}}+\frac{4}{3 \sqrt{2}{k_{2}}^{3}}\left|w_{3}-w_{3}{ }^{*}\right|\left|\delta_{3}\right|^{\frac{3}{2}} .
\end{aligned}
$$

Substituting the fact $\left|\omega_{j}-\omega_{j}^{*}\right| \leq \sqrt{2}\left|\delta_{j}\right|^{\frac{1}{2}}$ into (35), it yields

$$
V(\boldsymbol{s}, \boldsymbol{w}) \leq 2\left(1-q_{0} \operatorname{sign}\left(q_{0}(0)\right)\right)+\frac{4}{3{k_{2}}^{3}} \delta_{1}{ }^{2}+\frac{4}{3{k_{2}}^{3}} \delta_{2}{ }^{2}+\frac{4}{3 k_{2}{ }^{3}} \delta_{3}{ }^{2} .
$$

Consider the condition where $q_{0}(0) \geq 0$, one has $\operatorname{sign}\left(q_{0}(0)\right)=1$ and $\operatorname{sign}\left(q_{0}(0)\right) \geq q_{0}$. From (21) and (34) we can confirm that there exists a time $t^{*}<\infty$ such that $q_{0} \geq 0, \forall t \geq t^{*}$, after which the following equation holds

$$
1-q_{0} \operatorname{sign}\left(q_{0}(0)\right) \leq 1-q_{0}^{2}={q_{1}}^{2}+{q_{2}}^{2}+q_{3}^{2}, t \geq t^{*} .
$$


Combining (36) and (37), we have

$$
V(\boldsymbol{s}, \boldsymbol{w}) \leq 2\left({q_{1}}^{2}+{q_{2}}^{2}+{q_{3}}^{2}\right)+\frac{4}{3 k_{2}{ }^{3}}\left(\delta_{1}{ }^{2}+\delta_{2}{ }^{2}+\delta_{3}{ }^{2}\right), t \geq t^{*}
$$

Letting $\beta=\max \left\{2, \frac{4}{3 k_{2}{ }^{3}}\right\}$, it provides

$$
V(\boldsymbol{s}, \boldsymbol{w}) \leq \beta\left({q_{1}}^{2}+{q_{2}}^{2}+{q_{3}}^{2}\right)+\beta\left({\delta_{1}}^{2}+{\delta_{2}}^{2}+{\delta_{3}}^{2}\right), t \geq t^{*}
$$

Hence, using Lemma 4, we can obtain the following equation:

$$
\dot{V}(\boldsymbol{s}, \boldsymbol{w})+\frac{k_{3}}{\beta^{\frac{3}{4}}} V^{\frac{3}{4}}(s, \boldsymbol{w}) \leq 0,
$$

guaranteed by (34) and (39). Hence, it can be derived from Lemma 1 that the convergence time of the system states to the equilibrium $(1,0,0,0,0,0,0)$ is finite.

The same proof will not be repeated for the case $q_{0}(0)<0$, because the theorems and methods used are similar as for $q_{0}(0) \geq 0$. Thus, we get straightly to the conclusion that when $q_{0}(0)<0$, system $(7)$ can converge to the equilibrium $(-1,0,0,0,0,0,0)$ in finite time.

Remark 3: We have already proved that the angular velocity of the spacecraft $\boldsymbol{\omega}$ will converge to the virtual controller $\boldsymbol{\omega}^{*}$ in finite time. The quaternion $\boldsymbol{q}$ will also converge to the set $\left\{(1,0,0,0)^{T},(-1,0,0,0)^{T}\right\}$. Since the virtual controller is $\omega_{i}^{*}=-\operatorname{sign}\left(q_{0}(0)\right) k_{2}\left\lfloor q_{i}\right\rfloor^{\frac{1}{2}}, i=1,2,3$, it is obvious that the angular velocity of the spacecraft $\boldsymbol{\omega}$ will converge to $(0,0,0)^{T}$ in finite time synchronously.

Remark 4: As the control inputs $\boldsymbol{u}_{\boldsymbol{s}}(\boldsymbol{t})$ appear in the second derivative of the sliding variables, the effect of discontinuous control inputs on the attitude can be addressed substantially after two times of integral. According to [24], controller (33) can be regarded as SOSM controller because it guarantees the establishment of the SOSM $s_{i}=\dot{s}_{i}=0, i=0,1,2,3$.

It can be derived from the aforementioned analysis that the spacecraft attitude stabilization system (7) under the SOSM controller (33) is globally finite-time stable with respect to set $M$.

Remark 5: In practice, the discontinuous control inputs caused by the symbolic function in (33) may lead to chattering phenomenon. To avoid this, the continuous function $\frac{|s|}{|s|+\phi}$ can be used as a substitute for the discontinuous symbolic function $\operatorname{sign}(s)$. Although the stability of the system under the continuous control inputs degrades from finite-time stable to finite-time 
boundness, the boundary can be reduced to a tolerable range by choosing a appropriately small $\phi$. The continuous controller is of the form

$$
u_{s i}=-k_{1} \frac{\left|\delta_{i}\right|}{\left|\delta_{i}\right|+\phi}, i=1,2,3,
$$

where $k_{1}, k_{2}, k_{3}$ are the same as in (33), $\phi$ is chosen as a small positive constant. The effectiveness of the controller will be verified by simulations in Section 4 .

\section{Simulation results}

In this section, the simulation results are presented in the form of figures and tables. Choosing the inertia matrix as

$$
\boldsymbol{J}=\left[\begin{array}{lll}
3 & 1 & 1 \\
1 & 2 & 0 \\
1 & 0 & 1
\end{array}\right]
$$

The controllers involved in the following comparison section are designed as follows:

The integral SOSM (ISOSM) controller is designed as [37]

$$
\boldsymbol{u}(\boldsymbol{t})=\boldsymbol{B}^{-\mathbf{1}}(\boldsymbol{q}, \boldsymbol{\omega})\left[-\boldsymbol{A}(\boldsymbol{q}, \boldsymbol{\omega})-k_{1}\left\lfloor\boldsymbol{q}_{\boldsymbol{v}}\right\rceil^{\alpha_{1}}-k_{2}\left\lfloor\dot{\boldsymbol{q}}_{\boldsymbol{v}}\right\rceil^{\alpha_{2}}-\mu \boldsymbol{s}-\lambda \operatorname{sign}(\boldsymbol{s})\right],
$$

where $\boldsymbol{s}(\boldsymbol{t})=\dot{\boldsymbol{q}}_{\boldsymbol{v}}(\boldsymbol{t})-\int_{0}^{t}\left(-k_{1}\left\lfloor\left.\boldsymbol{q}_{\boldsymbol{v}}\right|^{\alpha_{1}}-k_{2}\left\lfloor\dot{\boldsymbol{q}}_{\boldsymbol{v}}\right\rceil^{\alpha_{2}}\right) d \tau\right.$ is the integral sliding mode surface.

The global finite time control (GFTC) controller is designed as [45]

$$
u_{s i}=-k_{1}\left(\omega_{i}^{p}+\operatorname{sign}\left(q_{0}(0)\right) k_{2}^{p} q_{i}\right)^{\frac{2}{p-1}}, i=1,2,3,
$$

where $k_{1}, k_{2}$ are all positive constants.

\subsection{Validation of set stability}

In order to verify the global finite-time stability mentioned above, the RPSOSM algorithm proposed in this paper is simulated separately. The initial conditions of $\boldsymbol{q}, \boldsymbol{w}$ and $\boldsymbol{d}(\boldsymbol{t})$ are chosen as $\boldsymbol{q}(\mathbf{0})^{\boldsymbol{T}}=\left(q_{0}(0), q_{1}(0), q_{2}(0), q_{3}(0)\right)^{T}=(0.4,0.6,0.4, \sqrt{0.32})^{T}, \boldsymbol{\omega}(\mathbf{0})^{\boldsymbol{T}}=$ $\left(\omega_{1}(0), \omega_{2}(0), \omega_{3}(0)\right)^{T}=(0,0,0)^{T}, \boldsymbol{d}(\boldsymbol{t})^{\boldsymbol{T}}=\left(d_{1}, d_{2}, d_{3}\right)^{T}=(0,0,0)^{T}$. As the initial condition of $q_{0}$ satisfies $q_{0}(0) \geq 0$ here, combining with the results in comparison section where $q_{0}(0)$ is chosen as $q_{0}(0)<0$, the global stability under the designed controller can be verified. The simulation results are shown in Figs. 1-3. 


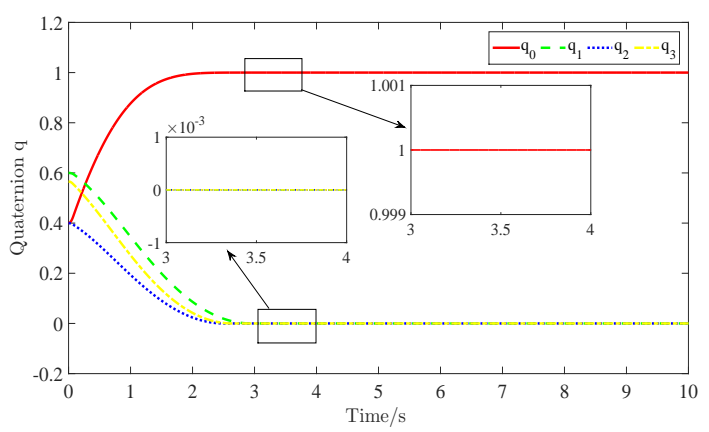

Fig. 1 Response curves of quaternion without disturbances.

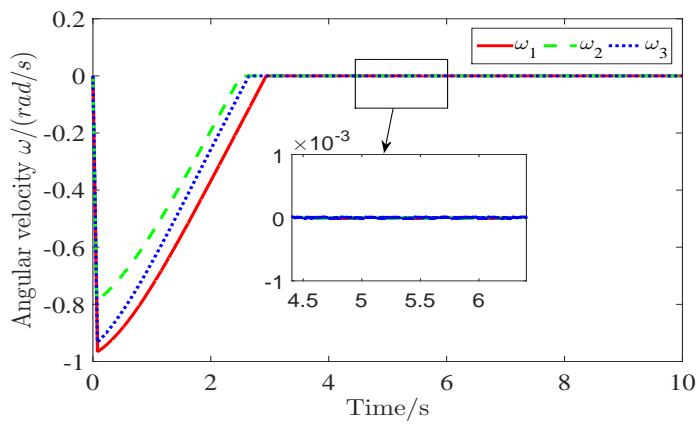

Fig. 2 Response curves of angular velocity $\omega$ without disturbances.
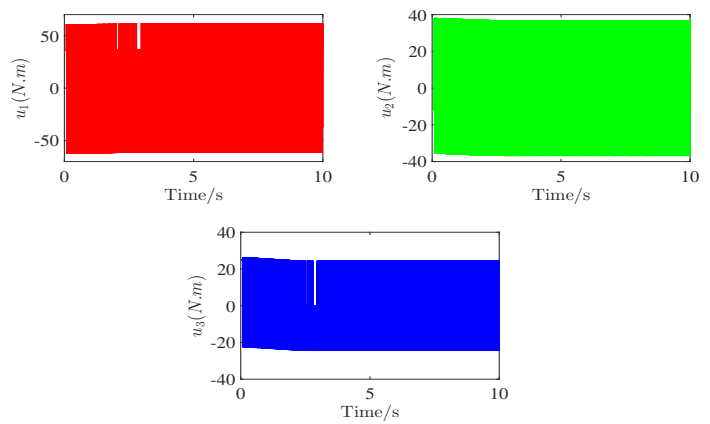

Fig. 3 Response curves of control torque without disturbances.

As is shown in Figs. 1-3, when $q_{0}(0)$ satisfies $q_{0}(0) \geq 0, q^{T}$ converges to $(1,0,0,0)^{T}$, which indicates that $(1,0,0,0)^{T}$ is one of the equilibria of the system. Also, though there exist discontinuous items in actual control input, the accuracy of the controller remains at a satisfying level. Therefore, we can 
reasonably claim that the influence of discontinuous control inputs on attitude accuracy is dramatically addressed.

\subsection{Comparisons of the control performance}

In this section, the performance of the proposed RPSOSM algorithm (33), the ISOSM (42) algorism and the GFTC (43) algorism are compared in the absence/presence of disturbances.

The comparisons are divided into two cases: Case 1 considers the system with disturbances, while Case 2 is the case when the system subjects to sinusoidal disturbances. In Case 1, the initial conditions of $\boldsymbol{q}, \boldsymbol{\omega}$ and $\boldsymbol{d}(\boldsymbol{t})$ are chosen as $\boldsymbol{q}(\mathbf{0})^{\boldsymbol{T}}=\left(q_{0}(0), q_{1}(0), q_{2}(0), q_{3}(0)\right)^{T}=(-0.2,0.6,-0.4, \sqrt{0.44})^{T}, \boldsymbol{\omega}(\mathbf{0})^{\boldsymbol{T}}=$ $\left(\omega_{1}(0), \omega_{2}(0), \omega_{3}(0)\right)^{T}=(0,0,0)^{T}, \boldsymbol{d}(\boldsymbol{t})^{\boldsymbol{T}}=\left(d_{1}, d_{2}, d_{3}\right)^{T}=(0,0,0)^{T}$. In Case 2, the disturbances $\boldsymbol{d}(\boldsymbol{t})$ are selected as $\boldsymbol{d}(\boldsymbol{t})^{\boldsymbol{T}}=\left(d_{1}, d_{2}, d_{3}\right)^{T}=$ $(\sin (10 t), \sin (20 t), \sin (30 t))^{T}$. Noting that in order to ensure the fairness of comparisons, suitable parameters are adjusted to ensure the performance of ISOSM and GFTC, and the control inputs are uniformly limited to 65N.m. The parameters of the above-mentioned controllers are shown in Table. 1 . The simulation results of Case 1 and Case 2 are shown in Figs. 4-6 and Figs. 7-9, respectively.
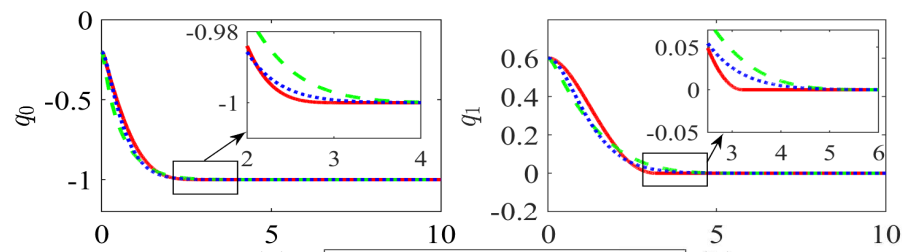

(a) -RPSOSM - -ISOSM ------GFTC (b)

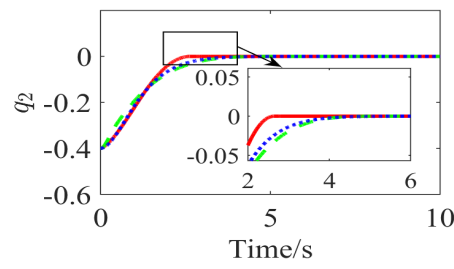

(c)

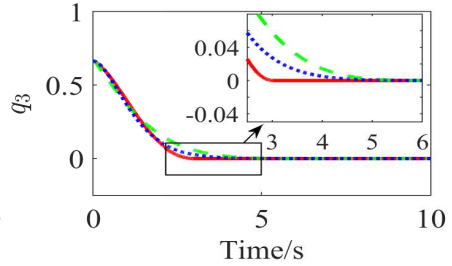

(d)

Fig. 4 Response curves of quaternion without disturbances. (a) $q_{0}$. (b) $q_{1}$. (c) $q_{2}$. (d) $q_{3}$. 


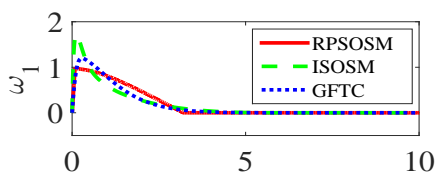

(a)

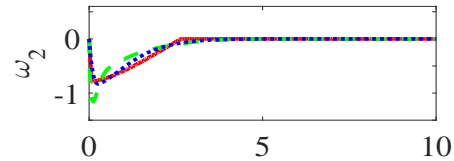

(c)

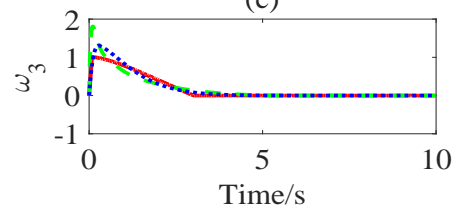

(e)

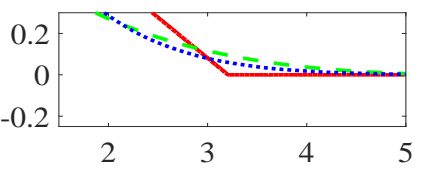

(b)

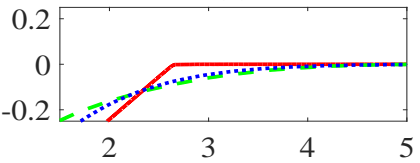

(d)

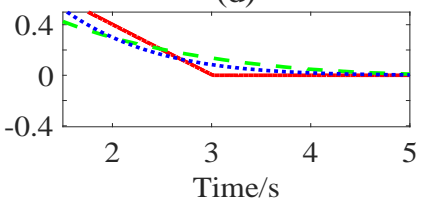

(f)

Fig. 5 Response curves of angular velocity without disturbances. (a) $\omega_{1}$. (b) details of $\omega_{1}$. (c) $\omega_{2} \cdot\left(\right.$ d) details of $\omega_{2}$. (e) $\omega_{3}$. (f) details of $\omega_{3}$.

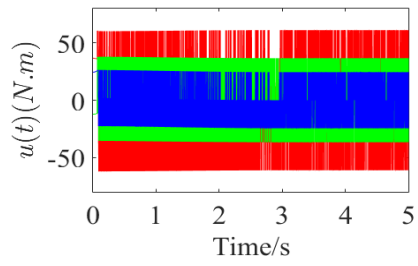

(a)

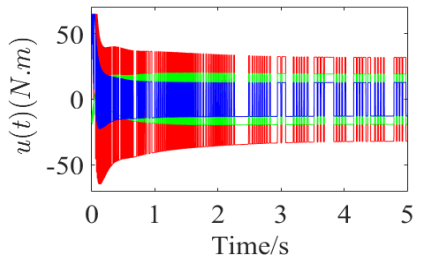

(b)

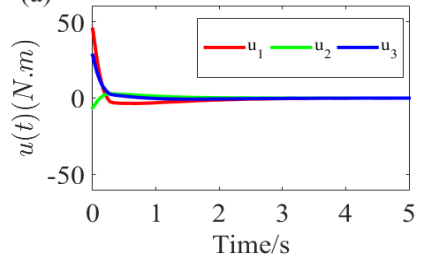

(c)

Fig. 6 Response curves of control torque without disturbances. (a) RPSOSM (33). (b) ISOSM (42). (c) GFTC (43).

Table 1 Controller parameters

\begin{tabular}{c|c}
\hline Controllers & Parameters \\
\hline RPSOSM $(33)$ & $k_{1}=30, k_{2}=1.5, k_{3}=0.01$ \\
\hline ISOSM $(42)$ & $k_{1}=6, k_{2}=9, \alpha_{1}=0.5, \alpha_{2}=2 / 3, \lambda=3.2, \mu=0.5$ \\
\hline GFTC $(43)$ & $k_{1}=35, k_{2}=1.7, k_{3}=0.01, p=19 / 17$ \\
\hline
\end{tabular}


When $q_{0}(0)$ is selected as $q_{0}<0, \boldsymbol{q}^{\boldsymbol{T}}$ converges to $(-1,0,0,0)^{T}$. Therefore, $(-1,0,0,0)^{T}$ is also the equilibrium of the system, thus verifying the global stability of the designed controller. In the absence of disturbance, RPSOSM has a faster convergence speed than ISOSM and GFTC, and the discontinuity existing in the control does not affect the accuracy of the controller.

Subsequently, when the system is subject to sinusoidal disturbances of different frequencies, it is obvious that RPSOSM still owns a faster convergence rate and higher stable precision than ISOSM and GFTC. The quantitative analysis of the simulation results is shown in Table. 2.
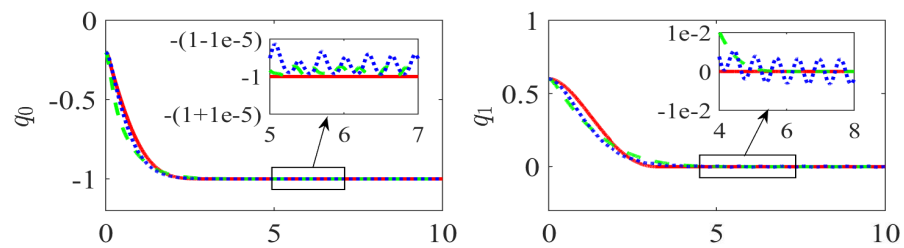

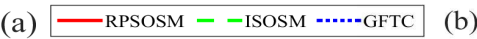

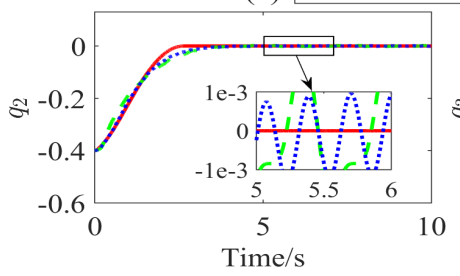

(c)

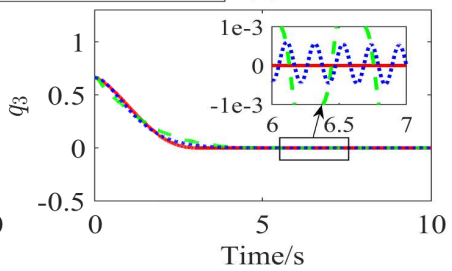

(d)

Fig. 7 Response curves of quaternion with disturbances. (a) $q_{0}$. (b) $q_{1}$. (c) $q_{2}$. (d) $q_{3}$.

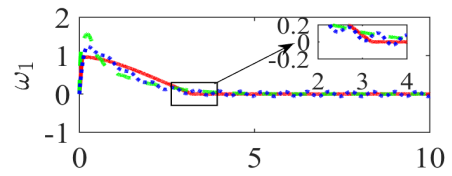

(a)

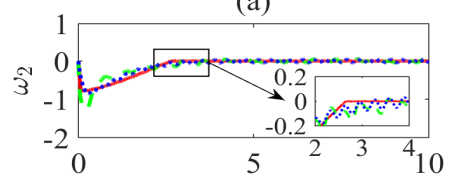

(c)

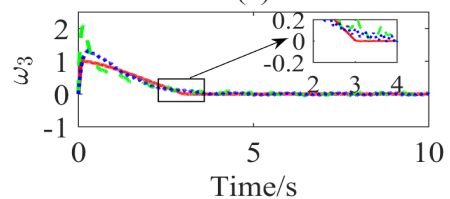

(e)

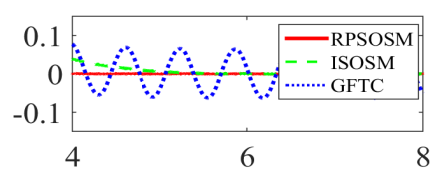

(b)

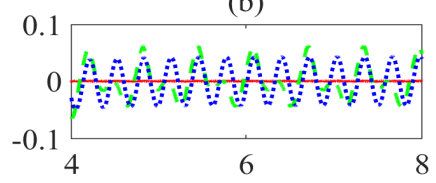

(d)

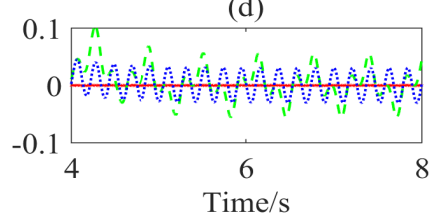

(f)

Fig. 8 Response curves of angular velocity with disturbances. (a) $\omega_{1}$. (b) details of $\omega_{1}$. (c) $\omega_{2}$. (d) details of $\omega_{2}$. (e) $\omega_{3}$. (f) details of $\omega_{3}$. 


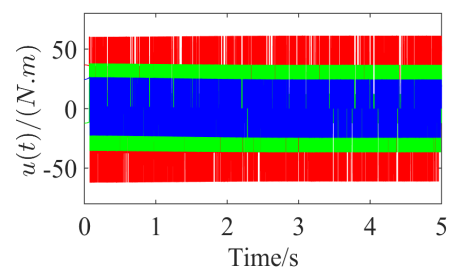

(a)

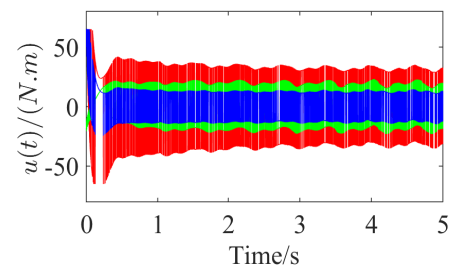

(b)

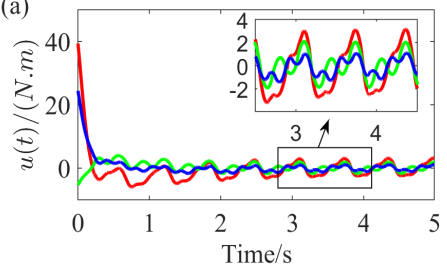

(c)

Fig. 9 Response curves of control torque with disturbances. (a) RPSOSM (33). (b) ISOSM (42).(c) GFTC (43).

\subsection{Validation of the continuous controller}

In this part, the proposed continuous controller (41) is simulated to verify its effectiveness. Since the main difference between the controller (41) and controller (33) is the disturbance rejection abilities, the simulation is carried out with disturbances. The parameters of controller (41) are the same with controller (33) and the extra parameter $\phi$ is chosen as 0.05 . With the same disturbances as that in the comparison section, the simulation results are shown in Figs. 10-12.

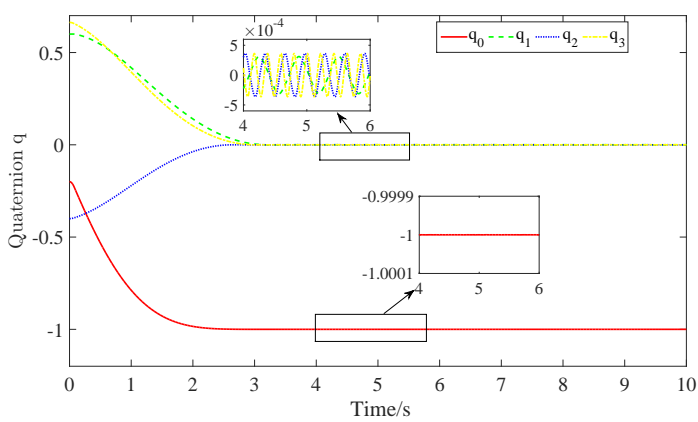

Fig. 10 Response curves of quaternion under continuous controller (41). 


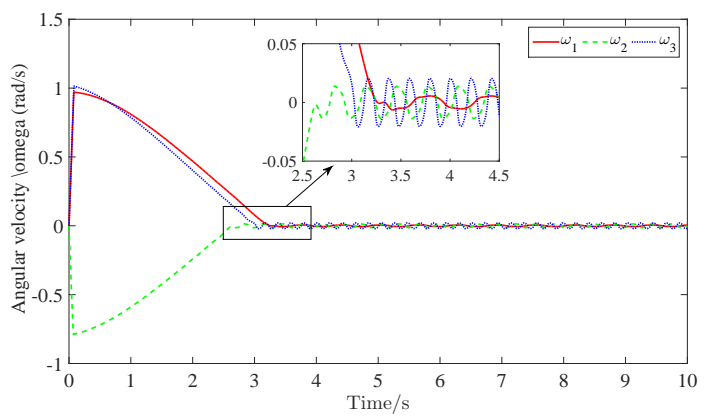

Fig. 11 Response curves of angular velocity $\omega$ under continuous controller (41).
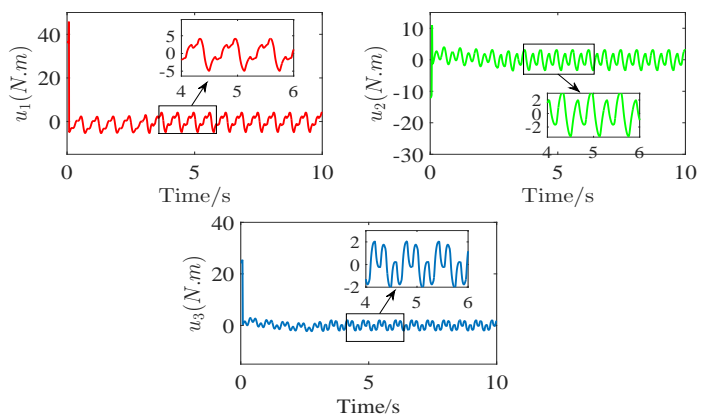

Fig. 12 Response curves of control torque under continuous controller (41).

Table 2 Performance comparison

\begin{tabular}{cccc}
\hline \multirow{2}{*}{ Test cases } & Controllers & \multicolumn{2}{c}{ Performance Indices } \\
\cline { 2 - 4 } & & $\mathrm{CT}(\mathrm{s})^{1}$ & $\mathrm{QE}^{2}$ \\
\hline \multirow{3}{*}{ Case 1 (without disturbances) } & $\operatorname{RPSOSM}(33)$ & 2.981 & 0 \\
\cline { 2 - 4 } & $\operatorname{ISOSM}(42)$ & 4.551 & 0 \\
\cline { 2 - 4 } & $\operatorname{GFTC}(43)$ & 4.023 & 0 \\
\hline \multirow{2}{*}{ Case 2 (with disturbances) } & $\operatorname{RPOSM}(33)$ & 2.979 & 0 \\
\cline { 2 - 4 } & $\mathrm{ISOSM}(42)$ & 4.375 & $2.121 e-3$ \\
\cline { 2 - 4 } & GFTC(43) & 4.012 & $3.042 e-3$ \\
\cline { 2 - 4 } & Controller(41) & 2.994 & $3.911 e-4$ \\
\hline
\end{tabular}

${ }^{1} \mathrm{CT}$ (convergence time) denotes the time after which $\left|q_{i}-q_{i}{ }^{*}\right|<5 e-3, i=0,1,2,3$ holds. ${ }^{2} \mathrm{QE}$ (quaternion error) denotes the maximum error of the quaternion after the convergence time, which is obtained by $Q E=\max \left|q_{i}-q_{i}^{*}\right|, i=0,1,2,3$. 


\section{Conclusion}

The global finite-time attitude stabilization of the rigid spacecraft by using SOSM control has been investigated in this article. A RPSOSM controller has been designed to obtain a finite-time result. The set stabilization idea has been applied to derive set stability for the system. The global finite-time stability has been proved based on a Lyapunov function. Simulation results reveal that the controller retain a satisfactory performance when dealing with bounded disturbances. Our future work is to further eliminate the discontinuity in the control input of the SOSM controller by using HOSMC.

\section{Compliance with ethical standards.}

\section{Conflict of Interest}

The authors declare that they have no conflict of interest.

\section{Data Availability Statements}

The authors declare that all other data supporting the findings of this study are available within the article. Source data for figures are provided with the paper.

\section{Declarations}

We declare that we have no financial and personal relationships with other people or organizations that can inappropriately influence our work, there is no professional or other personal interest of any nature or kind in any product, service and/or company that could be construed as influencing the position presented in, or the review of, the manuscript entitled Global Finite-Time Set Stabilization of Spacecraft Attitude with Disturbances using Second-Order Sliding Mode Control.

\section{References}

[1] Joshi, S., Kelkar, A., Wen, J.: Robust attitude stabilization of spacecraft using nonlinear quaternion feedback. IEEE Transactions on Automatic Control 40(10), 1800-1803 (1995). https://doi.org/10.1109/9.467669

[2] Byrnes, C.I., Isidori, A.: On the attitude stabilization of rigid spacecraft. Automatica 27(1), 87-95 (1991). https://doi.org/10.1016/0005-1098(91) 90008-P

[3] Hu, Q., Li, B., Zhang, Y.: Robust attitude control design for spacecraft under assigned velocity and control constraints. ISA Transactions 52(4), 480-493 (2013). https://doi.org/10.1016/j.isatra.2013.03.003 
[4] Wen, T.Y., Kreutz-Delgado, K.: The attitude control problem. IEEE Transactions on Automatic Control 36(10), 1148-1162 (1991). https: //doi.org/10.1109/9.90228

[5] Show, L.L., Juang, J.C., Lin, C.T., Jan, Y.W.: Spacecraft robust attitude tracking design: Pid control approach. In: American Control Conference, pp. 1360-1365 (2002)

[6] Sari, N.N., Jahanshahi, H., Fakoor, M.: Adaptive fuzzy pid control srategy for spacecraft attitude control. International Journal of Fuzzy Systems 21(3), 769-781 (2019). https://doi.org/10.1007/s40815-018-0576-2

[7] Liu, Y., Zhang, T., Song, J., Liang, B.: Adaptive spacecraft attitude tracking controller design based on similar skew-symmetric structure. Chinese Journal of Aeronautics 23(2), 227-234 (2010). https://doi.org/10.1016/ S1000-9361(09)60209-0

[8] Sun, L., Zheng, Z.: Disturbance-observer-based robust backstepping attitude stabilization of spacecraft under input saturation and measurement uncertainty. IEEE Transactions on Industrial Electronics 64(10), 79948002 (2017). https://doi.org/10.1109/TIE.2017.2694349

[9] Qiao, J., Li, X., Xu, J.: A composite disturbance observer and $h_{\infty}$ control scheme for flexible spacecraft with measurement delay and input delay. Chinese Journal of Aeronautics 32(6), 1472-1480 (2019). https://doi.org/ 10.1016/j.cja.2018.10.013

[10] Wu, S., Wen, S.: Robust h-infinity output feedback control for attitude stabilization of a flexible spacecraft. Nonlinear Dynamics 84(1), 405-412 (2016). https://doi.org/10.1007/s11071-016-2624-5

[11] Luo, W., Chu, Y.-C., Ling, K.-V.: Inverse optimal adaptive control for attitude tracking of spacecraft. IEEE Transactions on Automatic Control 50(11), 1639-1654 (2005). https://doi.org/10.1109/TAC.2005.858694

[12] Horri, N., Palmer, P., Roberts, M.: Gain-scheduled inverse optimal satellite attitude control. IEEE Transactions on Aerospace and Electronic Systems 48(3), 2437-2457 (2012). https://doi.org/10.1109/TAES.2012. 6237602

[13] Dwyer III, T.A., Sira-Ramirez, H.: Variable-structure control of spacecraft attitude maneuvers. Journal of Guidance Control and Dynamics 11(3), 262-270 (1988). https://doi.org/10.2514/3.20303

[14] Xia, Y., Zhu, Z., Fu, M., Wang, S.: Attitude tracking of rigid spacecraft with bounded disturbances. IEEE Transactions on Industrial Electronics 58(2), 647-659 (2010). https://doi.org/10.1109/TIE.2010.2046611 
[15] Hu, Q., Xiao, L., Wang, C.: Adaptive fault-tolerant attitude tracking control for spacecraft with time varying inertia uncertainties. Chinese Journal of Aeronautics 32(3), 674-687 (2019). https://doi.org/10.1016/j.cja.2018. 12.015

[16] Seo, D., Akella, M.R.: High-performance spacecraft adaptive attitudetracking control through attracting-manifold design. Journal of Guidance Control and Dynamics 31(4), 884-891 (2008). https://doi.org/10.2514/1. 33308

[17] Bhat, S.P., Bernstein, D.S.: Finite-time stability of homogeneous systems. In: Proceedings of the American Control Conference, vol. 4, pp. 2513-2514 (1997)

[18] Bhat, S.P., Bernstein, D.S.: Finite-time stability of continuous autonomous systems. SIAM Journal on Control and Optimization 38(3), 751-766 (2000). https://doi.org/10.1137/S0363012997321358

[19] Du, H., Li, S.: Finite-time attitude stabilization for a spacecraft using homogeneous method. Journal of Guidance Control and Dynamics 35(3), 740-748 (2012). https://doi.org/10.2514/1.56262

[20] Zou, A., Kumar, K., de Ruiter, A.: Finite-time spacecraft attitude control under input magnitude and rate saturation. Nonlinear Dynamics 99(3), 2201-2217 (2019). https://doi.org/10.1007/s11071-019-05388-6

[21] Jiang, B., Li, C., Ma, G.: Finite-time output feedback attitude control for spacecraft using adding a power integrator technique. Aerospace Science and Technology 66, 342-354 (2017). https://doi.org/10.1016/j.ast.2017. 03.026

[22] Zuo, A., Kumar, K.: Finite-time attitude control for rigid spacecraft subject to actuator saturation. Nonlinear Dynamics 96(2), 1017-1035 (2019). https://doi.org/10.1007/s11071-019-04836-7

[23] Yu, X., Feng, Y., Man, Z.: Terminal sliding mode control - an overview. IEEE Open Journal of the Industrial Electronics Society $\mathbf{P P}(99)$, 1-1 (2020). https://doi.org/10.1109/OJIES.2020.3040412

[24] Levant, A.: Sliding order and sliding accuracy in sliding mode control. International Journal of Control 58(6), 1247-1263 (1993). https://doi. org/10.1080/00207179308923053

[25] Jin, E., Sun, Z.: Robust controllers design with finite time convergence for rigid spacecraft attitude tracking control. Aerospace Science and Technology 12(4), 324-330 (2008). https://doi.org/10.1016/j.ast.2007.08. 001 
[26] Hu, Q., B, L., Zhang, A.: Robust finite-time control allocation in spacecraft attitude stabilization under actuator misalignment. Nonlinear Dynamics 73(1-2), 53-71 (2013). https://doi.org/10.1007/ s11071-013-0766-2

[27] Huo, X., Hu, Q., Xiao, B.: Finite-time fault tolerant attitude stabilization control for rigid spacecraft. ISA Transactions 53(2), 241-250 (2014). https://doi.org/10.1016/j.isatra.2013.11.017

[28] Guo, B., Chen, Y.: Adaptive fast sliding mode fault tolerant control integrated with disturbance observer for spacecraft attitude stabilization system. ISA Transactions 94, 1-9 (2019). https://doi.org/10.1016/j. isatra.2019.04.014

[29] Cao, L., Xiao, B., Golestani, M.: Robust fixed-time attitude stabilization control of flexible spacecraft with actuator uncertainty. Nonlinear Dynamics 100, 3 (2020). https://doi.org/10.1007/s11071-020-05596-5

[30] Hu, Q., Niu, G.: Attitude output feedback control for rigid spacecraft with finite-time convergence. ISA Transactions 70, 173-186 (2017). https: //doi.org/10.1016/j.isatra.2017.07.023

[31] Li, B., Qin, K., Xiao, B., Yang, Y.: Finite-time extended state observer based fault tolerant output feedback control for attitude stabilization. ISA Transactions 91, 11-20 (2018). https://doi.org/10.1016/j.isatra.2019.01. 039

[32] Shao, S., Zong, Q., Tian, B., Wang, F.: Finite-time sliding mode attitude control for rigid spacecraft without angular velocity measurement. Journal of the Franklin Institute 354(12), 4656-4674 (2017). https://doi.org/10. 1016/j.jfranklin.2017.04.020

[33] Jiang, B., Hu, Q., Friswell, M.: Fixed-time attitude control for rigid spacecraft with actuator saturation and faults. IET Control Theory \& Applications 24(5), 1892-1898 (2016). https://doi.org/10.1109/TCST. 2016.2519838

[34] Chen, Q., Xie, S., Sun, M., He, X.: Adaptive nonsingular fixed time attitude stabilization of uncertain spacecraft. IEEE Transactions on Aerospace and Electronic Systems 54(6), 2937-2950 (2018). https://doi. org/10.1109/TAES.2018.2832998

[35] Zhu, Z., Xia, Y., Fu, M.: Adaptive sliding mode control for attitude stabilization with actuator saturation. IEEE Transactions on Industrial Electronics 58(10), 4898-4907 (2011). https://doi.org/10.1109/TIE.2011. 2107719 
[36] Esmaeilzadeh, S., Golestani, M., Mobayen, S.: Chattering-free faulttolerant attitude control with fast fixed-time convergence for flexible spacecraft. International Journal of Control Automation and Systems 19(2), 767-776 (2020). https://doi.org/10.1007/s12555-020-0043-3

[37] Tiwari, P.M., Janardhanan, S.U., Nabi, M.U.: Rigid spacecraft attitude control using adaptive integral second order sliding mode. Aerospace Science and Technology 42, 50-57 (2015). https://doi.org/10.1016/j.ast. 2014.11.017

[38] Tiwari, P.M., Janardhanan, S.U., Nabi, M.U.: Attitude control using higher order sliding mode. Aerospace Science and Technology 54, 108-113 (2016). https://doi.org/10.1016/j.ast.2016.04.012

[39] Lin, Y., Sontag, E.D., Yuan, W.: Recent results on lyapunov-theoretic techniques for nonlinear stability. In: Proceedings of the 1994 American Control Conference, vol. 2, pp. 1771-1775 (1994)

[40] Rouche, N., Habets, P., Laloy, M.: Stability Theory by Liapunovs Direct Method. Springer, New York, UK (1977)

[41] Bhat, S.P., Bernstein, D.S.: A topological obstruction to continuous global stabilization of rotational motion and the unwinding phenomenon. Systems and Control Letters 39(1), 63-70 (2000). https://doi.org/10.1016/ S0167-6911(99)00090-0

[42] Angeli, D.: Almost global stabilisation of the inverted pendulum via continuous state feedback. Automatica 37, 1103-1108 (2001). https://doi. org/10.1016/S0005-1098(01)00064-4

[43] Tiwari, P., Janardhanan, S., un-Nabi, M.: Spacecraft anti-unwinding attitude control using second-order sliding mode. Asian Journal of Control 20(1), 455-468 (2018). https://doi.org/10.1002/asjc.1601

[44] Hashemi, S., Pariz, N., Sani, S.: Observer-based adaptive hybrid feedback for robust global attitude stabilization of a rigid body. IEEE Transactions on Aerospace and Electronic Systems 57(3), 1919-1929 (2021). https: //doi.org/10.1109/TAES.2021.3050665

[45] Li, S., Ding, S., Li, Q.: Global set stabilisation of the spacecraft attitude using finite-time control technique. International Journal of Control 82(5), 822-836 (2009). https://doi.org/10.1080/00207170802342818

[46] Liao, X.: Mathematical Theory and Application of Stability. Company of Center China Normal University, Wuhan, China (1988) 
[47] Ding, S., Li, S., Zheng, W.X.: Nonsmooth stabilization of a class of nonlinear cascaded systems. Automatica 48(10), 2597-2606 (2012). https: //doi.org/10.1016/j.automatica.2012.06.060

[48] Qian, C., Lin, W.: A continuous feedback approach to global strong stabilization of nonlinear systems. IEEE Transactions on Automatic Control 46(7), 1061-1079 (2001). https://doi.org/10.1109/9.935058

[49] Hardy, G.H., Littlewood, J.E., Polya, G.: Inequalities. Cambridge University Press, Cambridge, UK (1952)

[50] Fragopoulos, D., Innocenti, M.: Stability considerations in quaternion attitude control using discontinuous lyapunov functions. IEE Proceedings - Control Theory \& Applications 151(3), 253-258 (2004). https: //doi.org/10.1049/ip-cta:20040311

[51] Kane, T.R., Likins, P.W., Levinson, D.A.: Spacecraft dynamics. AIAA Journal 21(6), 928-928 (1983). https://doi.org/10.1115/1.3167078

[52] Pisacane, V.L.: Fundamentals of Space Systems. Oxford University Press, Pisacane and Robert C. Moore. New York (1994)

[53] Ding, S., Li, S.: Second-order sliding mode controller design subject to mismatched term. Automatica 77, 388-392 (2017). https://doi.org/10. 1016/j.automatica.2016.07.038 